\title{
The Lagrangian of self-dual gravitational field as a limit of the SDYM Lagrangian
}

\author{
Jerzy F. Plebański and Maciej Przanowskił \\ Department of Physics \\ Centro de Investigación y de Estudios Avanzados del IPN \\ Apartado Postal 14-740, México 07000, D.F., México
}

\begin{abstract}
The action for the $\operatorname{su}(\mathrm{N})$ SDYM equations is shown to give in the limit $N \rightarrow \infty$ the action for the sixdimensional version of the second heavenly equation. The symmetry reductions of this latter equation to the well known equations of self-dual gravity are given. The Moyal deformation of the heavenly equations are also considered.
\end{abstract}

PACS:04.20.Cv, 11.15.-q

\footnotetext{
${ }^{1}$ E-mail address: pleban@fis.cinvestav.mx

${ }^{2}$ Permanent address: Institute of Physics, Technical University of Lódź, Wólczańska 219, 93-005 Lódź, Poland
} 
Recently, a great deal of interest has been devoted to symmetry reductions of the SDYM equations to integrable equations of mathematical physics [116]. In particular many works concern the symmetry reductions of the SDYM equations to the self-dual gravity equations [6-16].

In our previous works we have found the general form of the $\mathrm{su}(\mathrm{N}) \mathrm{SDYM}$ equations which in the limit $\mathrm{N} \rightarrow \infty$ gives the sdiff $\left(\Sigma^{2}\right) \cong s u(\infty)$ SDYM equation in the form the second heavenly equation in six dimensions $[17,18]$. Then we have shown that the symmetry reductions of that equation lead to the well known heavenly equations of self-dual gravity.

We were also able to obtain the natural Moyal deformation of the heavenly equations. This deformation has been previously found by Strachan $[12,19]$ and Takasaki [20].

In the present work we are going to show that the limiting process $N \rightarrow \infty$ leading from $\mathrm{su}(\mathrm{N})$ SDYM equations to the heavenly equations can be also well defined on the action level. Similar considerations enable one to find the action for the Moyal deformation of the sixdimensional version of the second heavenly equation.

We deal with the su(N) SDYM equations in the flat 4-dimensional real, simply connected flat manifold $V \subset R^{4}$ of the metric

$$
d s^{2}=2\left(d x \otimes_{s} d \tilde{x}+d y \otimes_{s} d \tilde{y}\right)
$$

where $x, y, \tilde{x}, \tilde{y}$ are null coordinates on $V$ and $\otimes_{s}$ denotes the symmetrized tensor product, i.e., $d x \otimes_{s} d \tilde{x}=\frac{1}{2}(d x \otimes d \tilde{x}+d \tilde{x} \otimes d x)$. The coordinates $(x, y, \tilde{x}, \tilde{y})$ are chosen in such a manner that the $\mathrm{su}(\mathrm{N})$ SDYM equations read $[21]$

$$
\begin{aligned}
F_{x y} & =0 \\
F_{\tilde{x} \tilde{y}} & =0 \\
F_{x \tilde{x}}+F_{y \tilde{y}} & =0
\end{aligned}
$$

where, as usually, $F_{\mu \nu} \in \mathrm{su}(\mathrm{N}) \otimes C^{\infty}(V), \mu, \nu \in\{x, y, \tilde{x}, \tilde{y}\}$, stands for the Yang-Mills field tensor.

Then, in terms of the Yang-Mills potentials $A_{\mu} \in \mathrm{su}(\mathrm{N}) \otimes C^{\infty}(V)$ which define $F_{\mu \nu}$ according to the well known formula.

$$
F_{\mu \nu}=\left[\partial_{\mu}+A_{\mu}, \partial_{\nu}+A_{\nu}\right]
$$


one rewrites the equations (2.a, b, c) as follows

$$
\begin{aligned}
\partial_{x} A_{y}-\partial_{y} A_{x}+\left[A_{x}, A_{y}\right] & =0 \\
\partial_{\tilde{x}} A_{\tilde{y}}-\partial_{\tilde{y}} A_{\tilde{x}}+\left[A_{\tilde{x}}, A_{\tilde{y}}\right] & =0 \\
\partial_{x} A_{\tilde{x}}-\partial_{\tilde{x}} A_{x}+\partial_{y} A_{\tilde{y}}-\partial_{\tilde{y}} A_{y}+\left[A_{x}, A_{\tilde{x}}\right]+\left[A_{y}, A_{\tilde{y}}\right] & =0
\end{aligned}
$$

Eq. (4.a) implies that the potentials $A_{x}$ and $A_{y}$ are of the pure gauge form i.e., there exists an $\mathrm{SU}(\mathrm{N})$-valued function $g$ such that

$$
A_{x}=g^{-1} \partial_{x} g \text { and } A_{y}=g^{-1} \partial_{y} g .
$$

Therefore one can choose the gauge such that

$$
A_{x}=0 \text { and } A_{y}=0
$$

Henceforth we assume that this last condition holds. Consequently, Eqs. (4.a, b, c), under the condition (6), read

$$
\begin{aligned}
\partial_{\tilde{x}} A_{\tilde{y}}-\partial_{\tilde{y}} A_{\tilde{x}}+\left[A_{\tilde{x}}, A_{\tilde{y}}\right] & =0 \\
\partial_{x} A_{\tilde{x}}-\partial_{y} A_{\tilde{y}} & =0
\end{aligned}
$$

From (7.b) it follws that there exists an su(N)-valued function $\theta$ such that

$$
A_{\tilde{x}}=-\partial_{y} \theta \text { and } A_{\tilde{y}}=\partial_{x} \theta
$$

Substituting (8) into (7.a) one gets

$$
\begin{gathered}
\partial_{x} \partial_{\tilde{x}} \theta+\partial_{y} \partial_{\tilde{y}} \theta+\left[\partial_{x} \theta, \partial_{y} \theta\right]=0, \\
\theta \in \operatorname{su}(N) \otimes C^{\infty}(V) .
\end{gathered}
$$

Eq. (9) is equivalent to the $\operatorname{su}(\mathrm{N})$ SDYM equations (2.a, b, c).

Now, straightforward calculations show that Eq. (9) can be derived from the following least action principle 


$$
\begin{gathered}
\delta S=0, \quad S=\int_{V} \mathcal{L} d v, \quad d v=d x d y d \tilde{x} d \tilde{y} \\
\mathcal{L}:=\frac{(2 \pi)^{4}}{N^{3}} \operatorname{Tr}\left\{\frac{1}{3} \theta\left[\partial_{x} \theta, \partial_{y} \theta\right]-\frac{1}{2}\left(\left(\partial_{x} \theta\right)\left(\partial_{\tilde{x}} \theta\right)+\left(\partial_{y} \theta\right)\left(\partial_{\tilde{y}} \theta\right)\right)\right\}
\end{gathered}
$$

Thus the Lagrangian $\mathcal{L}$ defined by (10) can be considered to be the Lagrangian for the SDYM field.

Now we let $\mathrm{N}$ tend to infinity.

Thus we arrive at the $\mathrm{su}(\infty)$ algebra. It is well known that [22-26]

$$
\text { su }(\infty) \cong \operatorname{sdiff}\left(\Sigma^{2}\right) \cong \text { the Poisson algebra on } \Sigma^{2}
$$

where $\Sigma^{2}$ is a 2 -dimensional real manifold.

Employing the results of Refs [23, 24], where the case of $\Sigma^{2}$ being the 2 -torus has been considered, one can quickly find that in order to obtain the $N \rightarrow \infty$ limit of the action (10) we can proceed as follows: We consider $\theta$ to be the function on $V \times \Sigma^{2}$ i.e., $\theta=\theta(x, y, \tilde{x}, \tilde{y}, p, q)$ where $(p, q)$ are the coordinates on $\Sigma^{2}$. Moreover, we make the following substitutions

$$
\begin{aligned}
{[\cdot, \cdot] } & \rightarrow\{\cdot, \cdot\}_{P} \\
\frac{(2 \pi)^{4}}{N^{3}} T_{r}(\ldots) & \rightarrow-\int_{\Sigma^{2}}(\ldots) d p d q,
\end{aligned}
$$

where $\{\cdot, \cdot\}_{P}$ stands for the Poisson bracket

$$
\left\{f_{1}, f_{2}\right\}_{P}:=\frac{\partial f_{1}}{\partial q} \frac{\partial f_{2}}{\partial p}-\frac{\partial f_{1}}{\partial p} \frac{\partial f_{2}}{\partial q}
$$

for any $f_{1}=f_{1}(x, y, \tilde{x}, \tilde{y}, p, q)$ and $f_{2}=f_{2}(x, y, \tilde{x}, \tilde{y}, p, q)$.

Thus the action $S$ defined by (10) is brought to the following form

$$
\begin{gathered}
S \rightarrow S_{\infty}=\int_{V \times \Sigma^{2}}\left\{-\frac{1}{3} \theta\left\{\partial_{x} \theta, \partial_{y} \theta\right\}_{P}+\frac{1}{2}\left(\left(\partial_{x} \theta\right)\left(\partial_{\tilde{x}} \theta\right)\right.\right. \\
\left.\left.+\left(\partial_{y} \theta\right)\left(\partial_{\tilde{y}} \theta\right)\right)\right\} d v d p d q .
\end{gathered}
$$

This shows that we now deal with the Lagrangian in a 6-dimensional space $V \times \Sigma^{2}$ 


$$
\mathcal{L}_{\infty}=-\frac{1}{3} \theta\left\{\partial_{x} \theta, \partial_{y} \theta\right\}_{P}+\frac{1}{2}\left(\left(\partial_{x} \theta\right)\left(\partial_{\tilde{x}} \theta\right)+\left(\partial_{y} \theta\right)\left(\partial_{\tilde{y}} \theta\right)\right)
$$

The similar Lagrangian in four dimensions was first considered in [31].

It is an easy matter to prove that the Euler-Lagrange equation for $\mathcal{L}_{\infty}$ reads

$$
\theta_{x \tilde{x}}+\theta_{y \tilde{y}}+\left\{\theta_{x}, \theta_{y}\right\}_{P}=0
$$

where we use the obvious notation $\theta_{x}:=\partial_{x} \theta, \theta_{x \tilde{x}}:=\partial_{x} \partial_{\tilde{x}} \theta, \ldots$, etc.

Eq. (16) resembles very much the well known second heavenly equation [27] and we call it the sixdimensional version of the second heavenly equation.

This equation has been found in our previous works $[17,18]$ as the result of the $N \rightarrow \infty$ limit of Eqs. (7. a, b).

Here we show that the sixdimensional version of the second heavenly equation can be considered to be the $N \rightarrow \infty$ limit of the $\operatorname{su}(\mathrm{N})$ SDYM equations also on the action level.

Now we intend to present how the appropriate symmetry reductions of Eq. (16) lead to the heavenly equations (compare with [17, 18]).

(a) The first heavenly equation.

Let

$$
\begin{aligned}
& \theta=\theta^{\prime}-\frac{1}{2}(x \tilde{x}+y \tilde{y}) \\
& \theta_{x}^{\prime}=\theta_{\tilde{y}}^{\prime}, \quad \theta_{y}^{\prime}=-\theta_{\tilde{x}}^{\prime} .
\end{aligned}
$$

Then, the function $\theta^{\prime}$ is of the form

$$
\theta^{\prime}(x, y, \tilde{x}, \tilde{y}, p, q)=\Omega(x+\tilde{y}, \tilde{x}-y, p, q)
$$

and Eq. (16) is brought to the first heavenly equation [27]

$$
\Omega_{\tilde{x} q} \Omega_{\tilde{y} p}-\Omega_{\tilde{x} p} \Omega_{\tilde{y} q}=1
$$

(It is evident that the first heavenly equation can be also obtained when other symmetries are assumed, for example

$$
\left.\theta_{\tilde{x}}^{\prime}=0 \quad \text { and } \quad \theta_{\tilde{y}}^{\prime}=0\right) .
$$

(b) The second heavenly equation 
Here we assume the following symmetry

$$
\theta_{x}=\theta_{q} \quad \text { and } \quad \theta_{y}=\theta_{p}
$$

Consequently, $\theta$ takes the form

$$
\theta(x, y, \tilde{x}, \tilde{y}, p, q)=\Theta(x+q, y+p, \tilde{x}, \tilde{y})
$$

and now Eq. (16) is brought to the second heavenly equation [27]

$$
\Theta_{x \tilde{x}}+\Theta_{y \tilde{y}}+\Theta_{x x} \Theta_{y y}-\Theta_{x y}^{2}=0 .
$$

(c) Grant's equation.

Let now

$$
\theta_{x}=\theta_{\tilde{x}} \text { and } \theta_{\tilde{y}}=0
$$

Hence $\theta$ has the form

$$
\theta(x, y, \tilde{x}, \tilde{y}, p, q)=h(x+\tilde{x}, y, p, q)
$$

and Eq. (16) reads

$$
h_{x x}+h_{x q} h_{y p}-h_{x p} h_{y q}=0
$$

This is Grant's equation [28]

(d) The evolution form of the second heavenly equation.

Here we assume the symmetry

$$
\theta_{x}=-\theta_{\tilde{x}} \text { and } \theta_{y}=-\theta_{q}
$$

Thus

$$
\theta(x, y, \tilde{x}, \tilde{y}, p, q)=H(x-\tilde{x}, y-q, \tilde{y}, p)
$$

and, consequently, Eq. (16) is reduced to the evolution form of the second heavenly equation $[29,30]$

$$
H_{x x}-H_{y \tilde{y}}+H_{x y} H_{y p}-H_{x p} H_{y y}=0
$$

(e) Husain's equation 
This equation has been found by the reduction of the Ashtekar-JacobsonSmolin equations to the $\operatorname{siff} f\left(\Sigma^{2}\right)$ chiral field equations in two dimensions [13].

In our approach we assume the following symmetry

$$
\theta_{x}=\theta_{\tilde{x}} \quad \text { and } \quad \theta_{y}=\theta_{\tilde{y}}
$$

Therefore

$$
\theta(x, y, \tilde{x}, \tilde{y}, p, q)=\Lambda(x+\tilde{x}, y+\tilde{y}, p, q)
$$

and Eq. (16) takes the form of Husain's equation

$$
\Lambda_{x x}+\Lambda_{y y}+\Lambda_{x q} \Lambda_{y p}-\Lambda_{x p} \Lambda_{y q}=0
$$

To have a contact with some previous works $[29,31,32]$ we rewrite Eq. (16) in terms of 2 -spinors and then, in the differential forms langauge.

To this end put

$$
(x, y, \tilde{x}, \tilde{y}, p, q) \equiv\left(-p^{1},-p^{2}, q_{1}, q_{2}, \tilde{q}_{2}, \tilde{q}_{1}\right) .
$$

Hence, Eq. (16) reads now

$$
\frac{1}{2} \frac{\partial^{2} \theta}{\partial p^{A} \partial \tilde{q}_{B}} \frac{\partial^{2} \theta}{\partial p_{A} \partial \tilde{q}^{B}}+\frac{\partial^{2} \theta}{\partial p^{A} \partial q_{A}}=0, A, B=1,2,
$$

where the spinorial indices are to be manipulated according to the rule

$$
\begin{gathered}
\phi_{A}=\epsilon_{A B} \phi^{B}, \quad \phi^{A}=\epsilon^{B A} \phi_{B} \\
\left(\epsilon_{A B}\right):=\left(\begin{array}{cc}
0 & 1 \\
-1 & 0
\end{array}\right)=:\left(\epsilon^{A B}\right), \quad A, B,=1,2 .
\end{gathered}
$$

Then the Lagrangian $\mathcal{L}_{\infty}$ defined by (15) takes the form

$$
\mathcal{L}_{\infty}=-\frac{1}{6} \theta \frac{\partial^{2} \theta}{\partial p^{A} \partial \tilde{q}^{B}} \frac{\partial^{2} \theta}{\partial p_{A} \partial \tilde{q}_{B}}-\frac{1}{2} \frac{\partial \theta}{\partial p^{A}} \frac{\partial \theta}{\partial \tilde{q}_{A}} .
$$

We now denote

$$
\left(\theta_{x}, \theta_{y}, \theta_{\tilde{x}}, \theta_{\tilde{y}}, \theta_{p}, \theta_{q}\right) \equiv\left(s_{1}, s_{2}, r^{1}, r^{2}, \tilde{r}^{2}, \tilde{r}^{1}\right) .
$$


Consequently, by straightforward calculations one can show that in terms of differential forms the sixdimensional version of the heavenly equation (34) can be written as follows

$$
\begin{aligned}
d s^{A} \wedge d p_{A}+d r^{A} \wedge d q_{A}+d \tilde{r}^{A} \wedge d \tilde{q}_{A} & =0 \\
\left(d \tilde{q}_{A} \wedge d \tilde{q}^{A}+d s^{A} \wedge d q_{A}\right) \wedge d s^{B} \wedge d q_{B} \wedge d p^{C} \wedge d p_{C} & =0 . \\
d p^{A} \wedge d p_{A} \wedge d q^{B} \wedge d q_{B} \wedge d \tilde{q}^{C} \wedge d \tilde{q}_{C} & \neq 0
\end{aligned}
$$

Thus, to obtain from (38.a, b c) the second heavenly equation one assumes (see $(21))$

$$
s_{A}=\tilde{r}^{A}
$$

This leads to the equation

$$
\begin{gathered}
\frac{1}{2} \frac{\partial^{2} \Theta}{\partial p^{A} \partial p_{B}} \frac{\partial^{2} \Theta}{\partial p_{A} \partial p^{B}}+\frac{\partial^{2} \Theta}{\partial p^{A} \partial q_{A}}=0 \\
\Theta=\Theta\left(-p^{A}+\tilde{q}_{A}, q_{B}\right)
\end{gathered}
$$

which is exactly the second heavenly equation as written in terms of 2 -spinors $[29,31,32]$

To get the first heavenly equation we put

$$
s^{A}+\frac{1}{2} q^{A}=r^{A}-\frac{1}{2} p^{A} .
$$

Then from (38.a) one infers the existence of a function $\Omega$ such that

$$
\frac{\partial \Omega}{\partial p_{A}}=\frac{\partial \Omega}{\partial q_{A}}=r^{A}-\frac{1}{2} p^{A}=s^{A}+\frac{1}{2} q^{A}
$$

i.e., $\Omega$ is of the form

$$
\Omega=\Omega\left(q_{A}+p_{A}, \tilde{q}_{B}\right) .
$$

Consequently, (38.b) and (42) give the first heavenly equation [29, 31, 32]

$$
\frac{1}{2} \frac{\partial^{2} \Omega}{\partial q^{A} \partial \tilde{q}_{B}} \frac{\partial^{2} \Omega}{\partial q_{A} \partial \tilde{q}^{B}}+1=0 .
$$


Finally, from (41) and (42) we obtain the relation (compare with (17))

$$
\theta=\Omega+\frac{1}{2} p^{A} q_{A}
$$

Analogously one can find other heavenly equations in the spinorial form.

Now it is evident that the sixdimensional version of the second heavenly equation (34) implies the following conservation law

$$
\frac{\partial}{\partial p_{A}}\left(\frac{1}{2} \frac{\partial^{2} \theta}{\partial p^{A} \partial \tilde{q}_{B}} \frac{\partial \theta}{\partial \tilde{q}^{B}}-\frac{\partial \theta}{\partial q^{A}}\right)=0
$$

According to our philosophy this conservation law overlaps the hierarchy of conservation laws for the heavenly equations.

For example, in the case of the second heavenly equation, by (39), we have $-\frac{\partial \theta}{\partial p^{A}}=\frac{\partial \theta}{\partial \tilde{q}_{A}}$. Therefore, in this case (46) gives

$$
\frac{\partial}{\partial p_{A}}\left(\frac{1}{2} \frac{\partial^{2} \Theta}{\partial p^{A} \partial p_{B}} \frac{\partial \Theta}{\partial p^{B}}-\frac{\partial \Theta}{\partial q^{A}}\right)=0,
$$

where we substituted $\Theta$ in place of $\theta$ (see 40)).

On the other side in the case of the first heavenly equation, employing (42) and (45), one quickly finds that the conservation law (46) reads

$$
\frac{\partial}{\partial q_{A}}\left(\frac{\partial^{2} \Omega}{\partial q^{A} \partial \tilde{q}_{B}} \frac{\partial \Omega}{\partial \tilde{q}^{B}}+q_{A}\right)=0 .
$$

It is quite natural to expect that the conservation law (46) in six dimensions generates an infinite hierarchy of conservation laws in four dimensions when the heavenly equaitons are assumed to hold. In order to prove this statement and also to find the relation of our approach with the previous works by Boyer and one of us (J.F.P) [29, 32] and by Strachan [33] we should first find the general theory of symmmetry reduction of the sixdimensional version of the second heavenly equation to the heavenly equations. The work on this theory is in progress.

Finally, we are going to consider the Moyal deformation of Eq. (16). To this end we consier the SDYM equations (7. a, b) assuming that the potentials are now the self-adjoint operator-valued functions on $V \subset R^{4}$ acting in a Hilbert space $\mathcal{H}=L^{2}\left(R^{1}\right)$. Thus we now deal with the equations 


$$
\begin{aligned}
\partial_{\tilde{x}} \hat{A}_{\tilde{y}}-\partial_{\tilde{y}} \hat{A}_{\tilde{x}}+\frac{1}{i \hbar}\left[\hat{A}_{\tilde{x}}, \hat{A}_{\tilde{y}}\right] & =0 \\
\partial_{x} \hat{A}_{\tilde{x}}+\partial_{y} \hat{A}_{\tilde{y}} & =0 \\
\hat{A}_{\tilde{x}}^{+}=\hat{A}_{\tilde{x}}, \hat{A}_{\tilde{y}}^{+} & =\hat{A}_{\tilde{y}} .
\end{aligned}
$$

Then from (49.b) we get

$$
\begin{gathered}
\hat{A}_{\tilde{x}}=-\partial_{y} \hat{\theta} \text { and } \hat{A}_{\tilde{y}}=\partial_{x} \hat{\theta}, \\
\hat{\theta}=\hat{\theta}(x, y, \tilde{x}, \tilde{y})=\hat{\theta}^{+} .
\end{gathered}
$$

Inserting (50) into (49.a) one obtains

$$
\partial_{x} \partial_{\tilde{x}} \hat{\theta}+\partial_{y} \partial_{\tilde{y}} \hat{\theta}+\frac{1}{i \hbar}\left[\partial_{x} \hat{\theta}, \partial_{y} \hat{\theta}\right]=0 .
$$

Straightforward calculations show that Eq. (51) can be derived from the following variational principle

$$
\begin{gathered}
\delta S^{(q)}=0, \quad S^{(q)}=\int_{V} \mathcal{L}^{(q)} d v \\
\mathcal{L}^{(q)}:=\operatorname{Tr}\left\{2 \pi \hbar\left[\frac{-1}{3 i \hbar} \hat{\theta}\left[\partial_{x} \hat{\theta}, \partial_{y} \hat{\theta}\right]+\frac{1}{2}\left(\left(\partial_{x} \hat{\theta}\right)\left(\partial_{\tilde{x}} \hat{\theta}\right)+\left(\partial_{y} \hat{\theta}\right)\left(\partial_{\tilde{y}} \hat{\theta}\right)\right]\right\}\right. \\
=2 \pi \hbar \sum_{j}<\psi_{j}\left|\left\{\frac{-1}{3 i \hbar} \hat{\theta}\left[\partial_{x} \hat{\theta}, \partial_{y} \hat{\theta}\right]+\frac{1}{2}\left(\left(\partial_{x} \hat{\theta}\right)\left(\partial_{\tilde{x}} \hat{\theta}\right)+\left(\partial_{y} \hat{\theta}\right)\left(\partial_{\tilde{y}} \hat{\theta}\right)\right)\right\}\right| \psi_{j}>,
\end{gathered}
$$

where $\left\{\mid \psi_{j}>\right\}_{j \in \mathcal{N}}$ constitutes an orthonormal basis in $\mathcal{H}$

$$
<\psi_{j}\left|\psi_{k}>=\delta_{j k}, \quad \sum_{j}\right| \psi_{j}><\psi_{j} \mid=\hat{1} .
$$

Employing the Weyl-Wigner-Moyal formalism [34-40] one can bring $\mathcal{L}^{(q)}$ to the following form 


$$
\begin{gathered}
\mathcal{L}^{(q)}=2 \pi \hbar \sum_{j} \int_{R^{2}}\left\{\frac{-1}{3} \theta *\left\{\partial_{x} \theta, \partial_{y} \theta\right\}_{M}+\frac{1}{2}\left(\left(\partial_{x} \theta\right) *\left(\partial_{\tilde{x}} \theta\right)\right.\right. \\
\left.\left.+\left(\partial_{y} \theta\right) *\left(\partial_{\tilde{y}} \theta\right)\right)\right\} \rho_{j}(p, q) d p d q
\end{gathered}
$$

where

$$
\theta=\theta(x, y, \tilde{x}, \tilde{y}, p, q):=\int_{-\infty}^{+\infty}<q-\frac{\xi}{2}|\hat{\theta}| q+\frac{\xi}{2}>\exp \left(\frac{i p \xi}{\hbar}\right) d \xi
$$

(the Weyl correspondence), and $\rho_{j}=\rho_{j}(p, q)$ denotes the Wigner function for $\mid \psi_{j}>$, i.e.,

$$
\rho_{j}=\rho_{j}(p, q):=\frac{1}{2 \pi \hbar} \int_{-\infty}^{+\infty}<q-\frac{\xi}{2}\left|\psi_{j}><\psi_{j}\right| q+\frac{\xi}{2}>\exp \left(\frac{i p \xi}{\hbar}\right) d \xi
$$

Moreover, the Moyal *-product is defined by

$$
\begin{aligned}
& *:=\exp \left(\frac{i \hbar}{2} \stackrel{\leftrightarrow}{\mathcal{P}}\right) \\
& \overleftrightarrow{\mathcal{P}}:=\frac{\overleftarrow{\partial}}{\partial q} \frac{\vec{\partial}}{\partial p}-\frac{\overleftarrow{\partial}}{\partial p} \frac{\vec{\partial}}{\partial q}
\end{aligned}
$$

and $\{., .\}_{M}$ stands for the Moyal bracket

$$
\begin{gathered}
\left\{f_{1}, f_{2}\right\}_{M}:=\frac{1}{i \hbar}\left(f_{1} * f_{2}-f_{2} * f_{1}\right)=\frac{2}{\hbar} f_{1} \sin \left(\frac{\hbar}{2} \stackrel{\leftrightarrow}{\mathcal{P}}\right) f_{2}, \\
f_{1}=f_{1}(x, y, \tilde{x}, \tilde{y}, p, q), f_{2}=f_{2}(x, y, \tilde{x}, \tilde{y}, p, q) .
\end{gathered}
$$

From (53) one quickly finds that

$$
\sum_{j} \rho_{j}=\frac{1}{2 \pi \hbar}
$$

Inserting (59) into (54) we get the action $\mathcal{S}^{(q)}$ to be of the form 


$$
\begin{gathered}
\mathcal{S}^{(q)}=\int_{V \times R^{2}}\left\{\frac{-1}{3} \theta *\left\{\partial_{x} \theta, \partial_{y} \theta\right\}_{M}+\frac{1}{2}\left(\left(\partial_{x} \theta\right) *\left(\partial_{\tilde{x}} \theta\right)\right.\right. \\
\left.\left.+\left(\partial_{y} \theta\right) *\left(\partial_{\tilde{y}} \theta\right)\right)\right\} d v d p d q .
\end{gathered}
$$

This action leads to the Moyal deformation of the sixdimensional version of the second heavenly equation

$$
\partial_{x} \partial_{\tilde{x}} \theta+\partial_{y} \partial_{\tilde{y}} \theta+\left\{\partial_{x} \theta, \partial_{y} \theta\right\}_{M}=0
$$

As

$$
\lim _{\hbar \rightarrow 0} f_{1} * f_{2}=f_{1} f_{2} \text { and } \lim _{\hbar \rightarrow 0}\left\{f_{1}, f_{2}\right\}_{M}=\left\{f_{1}, f_{2}\right\}_{P}
$$

one quickly finds that the sixdimensional version of the second heavenly equation (16) is the $\hbar \rightarrow 0$ limit of Eq. (61) and

$$
\lim _{\hbar \rightarrow 0} \mathcal{S}^{(q)}=\mathcal{S}_{\infty}
$$

Gathering all that one concludes tha the following relations hold

$\mathcal{O}$ SDYM EQUATIONS $\stackrel{\hbar \rightarrow 0}{\longrightarrow}$ HEAVENLY EQUATIONS $\stackrel{N \rightarrow \infty}{\longleftarrow}$ su $(\mathrm{N})$ SDYM EQUATIONS(where $\mathcal{O}$ denotes the set of the self-adjoint operators in $\mathcal{H}$ ).

\section{Remark:}

The Lagrangian (10) has been considered by Leznov [41] and then by Parkes [42]. Another SDYM Lagrangian has been proposed in [43, 44]. It has been written within the $\mathrm{J}$ formalism and we have not been able to find the analogy of the matrix $J$ in self dual gravity. We suppose that this analogy can be found when the Weyl-Wigner-Moyal formalism is used and then when the $\hbar \rightarrow 0$ limit is considered. The work on this problem is in progress. We are grateful to the referee for pointing out the papers [41-44] and for stating the question of uniqueress of the $N \rightarrow \infty$ limit for the SDYM Lagrangians.

We are indebted to H. García-Compeán for useful discussions. One of us (M.P.) is grateful to the staff of the Department of Physics at CINVESTAV for the warm hospitality.

This work is supported by CONACyT and CINVESTAV, México, D.F., México. 


\section{References}

[1] R.S. Ward, Nucl. Phys. B236 (1984) 381.

[2] R.S. Ward, Phil. Trans. R. Soc. Lond. A 315 (1985) 451.

[3] R.S. Ward, Multi-dimensional integrable systems, in: Field theory quantum gravity and strings, eds. H. J. de Vega and N.Sánchez, SpringerVerlag, Berlin (1986).

[4] L.J. Mason and G.A.J. Sparling, Phys Lett. A 137 (1989) 29.

[5] S. Chakravarty, M.J. Ablowitz and P.A. Clarkson, Phys. Rev. Lett. 65 (1989) 1085.

[6] M.J. Ablowitz and P.A. Clarkson, Solitons, nonlinear evolution equations and inverse scattering, LMS Lecture Notes Series vol. 149 (Cambridge Uniersity, Cambridge, 1991). See also the literature therein.

[7] L.J. Mason and E.T. Newman, Commun. Math. Phys. 121 (1989) 659.

[8] I. Bakas, Self-duality, integrable systems, W-algebras and all that, in:Non-linear fields: classical, random, semiclassical, eds. P.A. Garbaczewski and Z. Popowicz (World Scientific, Singapore, 1991).

[9] L.J. Mason, Twistor Newsletter 30 (1990) 14.

[10] S. Chakravarty, L.J. Mason and E.T.Newman, J. Math. Phys. 32 (1991) 1458.

[11] Q. Han Park, Int. J. Mod. Phys. A7 (1992) 1415.

[12] I.A.B. Strachan, Phys. Lett. B282 (1992) 63.

[13] V. Husain, Class. Quantum Gravity 11 (1994) 927.

[14] C. Castro, J. Math. Phys. 34 (1993) 681.

[15] J. F. Plebański, M. Przanowski and H. García-Compeán, Acta Phys. Pol. B25 (1994) 1079. 
[16] T. Ueno, Integrable field theories derived from 4D self- dual gravity, preprint, KHTP-95-08, hep-th/9508012.

[17] J. F. Plebański M. Przanowski, B. Rajca and J. Tosiek, Acta Phys. Pol. B26 (1995) 889.

[18] J. F. Plebański and M. Przanowski, The universal covering of heavenly equations via Weyl-Wigner-Moyal formalism, to appear.

[19] I.A.B. Strachan, The geometry of multidimensional integrable systems, preprint (1995).

[20] K. Takasaki, J. Geom. Phys. 14 (1994) 111.

K. Takasaki, J. Geom. Phys. 14 (1994)332.

[21] C.N. Yang. Phys. Rev. Lett. 38 (1977) 1377.

[22] J. Hoppe, Phys. Lett. B215 (1988) 706.

[23] D.B.Fairlie and C.K. Zachos, Phys. Lett. B224, (1989) 101.

[24] D.B. Fairlie, P. Fletcher and C.K. Zachos, J. Math. Phys. 31 (1990) 1088.

[25] E.G. Floratos, J. Iliopoulos and G. Tiktopoulos, Phys. Lett. B217 (1989) 285.

[26] P. Fletcher, Phys. Lett. B248 (1990) 323.

[27] J.F. Plebański, J. Math. Phys. 16 (1975) 2395.

[28] J.D.E. Grant, Phys. Rev. D 48 (1993) 2606.

[29] C.P. Boyer and J. F. Plebański, J. Math. Phys. 18 (1977) 1022.

[30] J.D. Finley III, J. F. Plebański, M. Przanowski and H. García-Compeán, Phys. Lett. A181 (1993) 435.

[31] C.P. Boyer, J.D. Finley III and J.F. Plebański, Complex general relativity, $\mathcal{H}$ and $\mathcal{H} \mathcal{H}$ spaces - a survey, in General relativity and gravitation, Einstein memorial volume, vol. 2, ed. A. Held (Plenum, New York, 1980) pp. 241-281. 
[32] C.P. Boyer and J.F. Plebański, J. Math. Phys. 26 (1985) 229.

[33] I.A.B. Strachan, Class. Quantum Gravity 10 (1993) 1417.

[34] H.Weyl, Z. Phys. 46 (1927) 1.

[35] E.P. Wigner, Phys. Rev. 40 (1932) 749.

[36] J.E. Moyal, Proc. Cambridge Phil. Soc. 45 (1949) 99.

[37] F. Bayen, M. Flato, C. Fronsdal, A. Lichnerowicz and D. Sternheimer, Ann. Phys. NY 111 (1978) 61; Ann. Phys. NY 111 (1978) 111.

[38] J.F. Plebański, Institute of Physics of Nicolaus Copernicus University, Toruń, preprint No 69 (1969).

[39] M. Hillery, R.F. O'Connell, M.O. Scully and E. P. Wigner, Phys. Rep. 106 (1984) 121.

[40] W.I. Tatarskij, Usp. Fiz. Nauk 139 (1983) 587.

[41] A.N. Leznov, Theor. M. Phys. 73 (1988) 1233.

[42] A. Parkes, Phys. Lett. B286 (1992) 265.

[43] V.P. Nair and J. Schiff, Phys. Lett. B 246 (1990) 423.

[44] B. Hou and L. Chao, Phys. Lett. B 298 (1993) 103. 